\title{
O EXAME FÍSICO E O ENFERMEIRO DE UTI
}

\author{
Miako Kimura* \\ Ana Maria Kazue Miyadahira* \\ Dina de Almeida Lopes Monteiro da Cruz** \\ Edna Ikumi Umebayashi Takahashi* \\ Katia Grillo Padilha* \\ Regina Marcı Cardoso de Sousa"*
}

KIMURA. M. et al. O exame físico e o enfermeiro de UTI. Rev. Esc. Enf. USP, v.28, n.2, p 156.70, ago. 1994.

Trata-se de um estudo realizado junto a 26 enfermeiros de UTI que teve como objetivos caracterızar a periodicıdade de realização dos itens componentes do exame físico, identificar em que momento o seu aprendizado foi mats significativo e levantar a opiniāo dos enfermeıros sobre a fase adequacia para "seu ensino. Os dados foram coletados mediante questionários enviados aos enfermeiros egressos dos Cursos de Especialização em Enfermagem em Cuidados Intensivos oferecidos pela Escola de Enfermagem da USP e que, na época da coleta de dados, trabalhavam em UTI. Os resultados permitıram concluir que de 45 itens apresentados, 31 (68,9\%) eram reali. zados frequentemente por mais de $50 \%$ dos enfermeiros. A pratica profissional foi considerada como o momento mais importante para o aprendizado do exame físico. Quanto à responsabilidade pelo seu ensino, constatou-se que 69,2\% dos enfermeiros atribuiram-na ao curso de graduaçāo.

UNITERMO: UTI - exame físico - ensino de enfermagem.

\section{INTRODUÇÃO}

$\mathrm{Na}$ unidade de Terapia Intensiva (UTI) a avaliação do paciente pelo enfermeiro assume fundamental importância tendo em vista que o objetivo principal do cuidado intensivo é a manutenção das funçōes vitais. O paciente apresenta, frequentemente, diferentes graus de comprometimento físico, que exigem do enfermeiro aguçada capacidade de observação, a fim de identificar e controlar precocemente qualquer instabilidade fisiológica.

Tais caracteristicas da assistência em UTI requerem deste profissional competência para o levantamento de dados do paciente, bem como habilidade

- Enfermeira. Doutor do Departamento de Enfermagem Médico-Cirúrgica da EEUSP

-. Enfermeira. Assistente Departamento de Enfermagem Médico-Cirirgica da EEUSP 
para tomar decisões frente a cada situação. A obtenção de dados envolve tanto técnicas de entrevista quanto de exame físico.

Nos últimos anos tem-se observado interesse crescente em consolidar a realização do exame físico como atividade própria do enfermeiro. Já na década de 70, autores como MORGAN; BATES (1971); BATES; LYNAUGH (1973, 1975) e JACKSON; MANTLE (1977) ressaltavam a importancia dessa atividade na ampliação de papéis na prática da enfermagem. Enfatizavam a necessidade de desenvolver nos profissionais habilidades necessárias à sua implementaçāo.

Em nosso meio, HORTA (1968) foi uma das pioneiras na proposiçāo de um roteiro de exame físico a ser realizado pelo enfermeiro, procurando sistematizar a observação do profissional na identificaçāo dos problemas de enfermagem na área física.

Os cursos de graduação em enfermagem vêm, desde então, incorporando o exame físico em seus conteúdos de ensino. Trabalhos e discussões em eventos científicos têm sido realizados em torno desse tema, possibilitando maior reflexāo sobre a importância do exame físico na qualificação da assistência de enfermagem.

Porém, apesar do tempo decorrido e dos esforços empreendidos, o que se pode observar é que ainda existem questionamentos a respeito da delimitação e da especificidade da coleta de dados em enfermagem. Um dos aspectos ainda nāo claramente definidos refere-se ao conteúdo necessário e suficiente para uma efetiva avaliação de enfermagem. Tal indefinição tem dificultado o direcionamento do ensino e da prática de uma avaliação mais voltada para o foco de ação específico de enfermagem.

Acreditando que a assistência de enfermagem em áreas específicas estabeleça demandas também especificas quanto ao conteúdo do exame físico, considera-se que a identificação dos itens componentes do exame físico que as enfermeiras têm realizado em UTI e de suas opiniōes quanto ao ensino desses coinponentes trarāo elementos que poderão ser úteis na formação de novos profissionais e nas discussōes acerca do assunto.

Diante do exposto, este estudo tem os seguintes objetivos:

- Caracterizar a periodicidade de realização dos componentes do exame físico pelos enfermeiros de UTI.

- Identificar a opinião dos enfermeiros de UTI quanto a fase de formação mais adequada para o ensino dos itens do exame físico.

- Identificar em que momento o aprendizado sobre o exame físico foi mais significativo para os enfermeiros de UTI.

\section{METODOLOGIA}

Este estudo foi realizado junto a enfermeiros egressos dos oito Cursos de Especialização em Enfermagem em Cuidados Intensivos oferecidos pela Escola de Enfermagem da Universidade de São Paulo no período de 1982 a 1991 e que, na época da coleta dos dados estavam trabalhando em UTI. 
A coleta de dados foi realizada pelas autoras durante o primeiro semestre de 1992. Foi elaborado um questionário (ANEXO I), previamente testado, contendo duas partes: uma referente a dados pessoais do profissional e a caracterização do seu local de trabalho; a outra referia-se às informaçōes específicas do estudo. Cada questionário foi acompanhado de uma carta esclarecendo a finalidade do estudo e de envelope selado para devolução.

A listagem de itens componentes do exame físico apresentada no instrumento de coleta de dados foi elaborada com base no trabalho de COLWELL; SMITH (1985); foram feitas algumas adaptaçōes na relação original, a fim de se obter uma composiçāo de exame físico geral que mais se aproximasse daquela praticada em nosso meio.

Cumpre esclarecer que os aspectos relativos à contribuição dos diferentes itens para as condutas de enfermagem e às barreiras ou dificuldades para a sua realizaçāo, também incluídos no instrumento de coleta de dados do presente estudo, deverão ser analisados em trabalho subseqüente.

Do total de 116 enfermeiros que concluíram os referidos cursos, obtevese previamente a informaçăo de que $\mathbf{4 3}$ não mais atuavam em UTI. Assim sendo, foram enviados 73 questionários. Destes, 42 retornaram, sendo 26 questionários respondidos, $11 \mathrm{com}$ a informaçāo de que esses enfermeiros não trabalhavam mais em UTI e $05 \mathrm{com}$ a informação de destinatário não localizado. Portanto para o cálculo de percentuais foi considerado o total de 26 respondentes.

\section{RESULTADOS E DISCUSSĀO}

\subsection{Caracterização da população}

A população do presente estudo foi constituída de 26 enfermeiros de UTI. Caracterizou-se como sendo predominantemente do sexo feminino $(92,2 \%)$. A idade variou de 24 a 41 anos encontrando-se a maioria da população $(53,8 \%)$ na faixa de 24 até 30 anos.

Considerando as informações relativas ao tempo de formatura e de trabalho desta população verificou-se que a maior parte dos enfermeiros está formada e trabalha em UTI de 5 a 10 anos, (52,0\% e 56,0\%, respectivamente). Por se tratar de uma população predominantemente jovem, justificam-se os percentuais obtidos na faixa de 2 a 5 anos tanto para o tempo de formatura $(19,3 \%)$ quanto para o tempo de trabalho em UTI $(30,8 \%)$. Em relação a esta última variável, encontrou-se apenas um enfermeira com mais de quinze anos de formada.

Quanto à caracterização dos locais de trabalho pôde-se verificar que a população do estudo encontrava-se trabalhando principalmente em UTIs gerais $(65,4 \%)$. Dos enfermeiros que trabalhavam em UTIs gerais, $46,2 \%$ atuavam exclusivamente com pacientes adultos e 19,2\% em locais que atendiam tanto crianças como adultos. Quanto ao tipo de atendimento, indepen- 
dente da UTI ser geral ou especializada, pôde-se constatar que a maioria trabalhava em locais que assistiam tanto a pacientes clínicos quanto a cirúrgicos.

Do total possivel de respostas $2 \%$ foram deixados em branco.

\subsection{Realização dos itens componentes do exame fisico}

Para a análise da frequêencia de realização dos itens componentes do exame físico, solicitou-se aos enfermeiros que indicassem se cada item é realizado freqüentemente, esporadicamente ou nunca.

A seguir serão apresentados os resultados considerando a periodicidade com que os itens do exame físico eram realizados por, no mínimo, $50 \%$ dos respondentes. Inicialmente serão apresentados os itens que eram realizados freqüentemente e a seguir os que eram realizados esporadicamente. Como não houve nenhum item que mais de 50\% dos enfermeiros tenha indicado como nunca realizado, serão discutidos, nessa categoria, os itens que tiveram maiores freqüências.

Ressalta-se que os percentuais foram calculados tendo por base o total de respondentes $(N=26)$.

QUADRO I - Itens componentes do exame fisico realizados freqüentemente pelos enfermeiros. Săo Paulo, 1992.

\begin{tabular}{|c|c|c|}
\hline Itens & $\underline{y}$ & 3 \\
\hline Respiraçào - frequència & 25 & 96,2 \\
\hline Respiração - ritmo & 25 & 96,2 \\
\hline Pulso arterial - ritmo & 25 & 96,2 \\
\hline Nível de consciència & 25 & 96.2 \\
\hline Membros - perfusão periférica & 25 & 96,2 \\
\hline Pulso arterial - frequêencia & 24 & 92,3 \\
\hline Pressāo arterial - & 24 & 92.3 \\
\hline Pele e fâneros - integridade & 24 & 92,3 \\
\hline Pele e fâneros - coloraçāo & 24 & 92.3 \\
\hline Pesquisa de edema & 24 & 92,3 \\
\hline Abdômen - ruidos hidroaéreos & 24 & 92.3 \\
\hline Temperatura & 23 & 88.5 \\
\hline Pele e fâneros - turgor & 23 & $\$ 8.5$ \\
\hline Respiração - ruidos (ausculta) & 22 & 84,6 \\
\hline Pressão venosa central & 22 & 34,6 \\
\hline Cabeça - olhos & 22 & $\$ 4.6$ \\
\hline Abdòmen - conformaçāo & 22 & 84.6 \\
\hline Membros - mobilidade & 22 & 84,6 \\
\hline Respiraçāo - amplitude & 21 & 80.8 \\
\hline Membros - sensibilidade & 21 & 80.8 \\
\hline Respiração - simetria & 20 & 76,9 \\
\hline Ausculta cardíaca - frequência & 20 & 76.9 \\
\hline Exame pupilar & 20 & 76.9 \\
\hline Cabeça - cavidade oral & 20 & 76,9 \\
\hline
\end{tabular}


QUADRO I - Itens componentes do exame fraco realizadas frequentemente pelos enfermeiros. São Paulo, 1992.

\begin{tabular}{lcr}
\hline Itens & $\mathrm{N}$ & $\%$ \\
Membros - rede vascular & 20 & $-6,9$ \\
\hline Ausculta cardiaca - ritmo & 19 & 73.1 \\
Genitália & 18 & 69.2 \\
Pressão arterial média & 15 & 57.7 \\
Tórax - conformaçāo & 15 & 57.7 \\
Pescoço - veias jugulares & 14 & 53.8 \\
Membros - musculatura & 14 & 53.8 \\
\hline
\end{tabular}

No total de 45 itens componentes do exame físico apresentados aos enfermeiros, $31(68,9 \%)$ eram realizados frequentemente por mass de $50 \%$ deles.

COLWELL; SMITH (1985), em estudo semelhante com enfermeiras de setores clínicos, encontraram que apenas cerca de um terço do total de trinta e seis itens do exame físico eram realizados diariamente por mais de $50 \%$ destas enfermeiras.

O primeiro aspecto que se pode analisar no QUADRO I é que nenhum item do exame físico é realizado freqüentemente por todos os enfermeiros deste estudo.

BATES (1982) divide o conteúdo do exame físico no seu livro de Propedêutica Médica nas seguintes áreas: pele, cabeça e pescoço; tórax e pulmōes: sistema cardiovascular; mamas e axilas; abdome; genitais; ânus, reto e próstata: sistema vascular periférico; sistema músculo-esquelético e sistema nervoso. Utilizando essas áreas como referência pode-se dizer que apenas as áreas referentes a mamas/ axilas e a ânus/ reto/ próstata não têm nenhum item contemplado pelos resultados no QUADRO I.

Faz-se necessário ressaltar que os itens referentes a essas áreas, embora sejam realizados freqüentemente ou esporadicamente por numero expressivo de enfermeiros, não foram citados pela maioria absoluta dos respondentes. Portanto, pode-se afirmar que, excetuando-se essas áreas, a maioria da população estudada realiza freqüentemente itens do exame físico relacionados a todas as demais áreas.

No QUADRO I figuram, com inaior frequência $(96,2 \%)$, itens relacionados ao risco iminente de vida ou a funçōes vitais básicas: respiratória. cardiovascular e neurológica. Essa relação pode justificar o fato desses itens serem realizados pela quase totalidade dos enfermeiros do estudo.

$E$ interessante observar que dentre os diferentes aspectos da respiração os enfermeiros parecem valorizar mais características como a frequência e o ritmo $(96,2 \%)$, do que a presença de ruidos $84,6 \%$ a amplitude $(80,8 \%)$ e a simetria $(76,4 \%)$. Seriam estes dados menos significativos no contexto de avaliaçào respiratória dos pacientes de UTI?

Observa-se também uma dissociação entre a obtenção do ritmo e frequêencia cardíacos pela palpação do pulso e pela ausculta cardíaca, esta menos utilizada do que aquela pelos enfermeiros deste estudo. Verifica-se assim, que 
apesar de constarem entre os itens freqüentemente realizados pelos enfermeiros, a ausculta tem sido menos utilizada no que se refere à avaliaçāo pulmonar e cardíaca. Já quanto à ausculta abdominal (pesquisa de ruídos hidroaéreos) a quase totalidade dos enfermeiros ( $92,3 \%)$, informou realizá-la.

Estes dados contrapõem-se aos obtidos por COLWELL; SMITH (1985), em estudo onde enfermeiras de unidades clínicas informaram utilizar diariamente muito mais a técnica de ausculta do que a de palpação.

QUADRO II - Itens do exame físico realizados esporadicamente pelos enfermeiros, Sāo Paulo, 1992.

\begin{tabular}{lcr}
\hline Itens & $\mathrm{N}$ & $\%$ \\
\hline Altura & 15 & 57.7 \\
Cabeça - orofaringe & 15 & 57,7 \\
Pescoço-pulso carotídeo & 15 & 57.7 \\
Cabeça-ouvidos & 14 & 53,8 \\
Cabeça - cavidade nasal & 14 & 53,8 \\
\hline
\end{tabular}

Dentre os itens esporadicamente realizados incluem-se a avaliação de orofaringe, ouvidos e cavidade nasal. Esses itens surgiram no estudo de COLWELL; SMITH (1985) como nunca realizados num percentual bastante expressivo $(49 \%)$. Os resultados encontrados parecem indicar que a avaliação dessas cavidades é realizada em situações mais específicas talvez quando outros indicadores apontem sua necessidade. No presente estudo como item esporadicamente realizado destaca-se ainda a altura, que por ser um dado estável no adulto pode ser obtida pela informaçăo do paciente, além de ser uma técnica de difícil realização em UTI pela própria condição física da clientela aí assistida. Em relação ao pulso carotídeo, pode-se justificar a sua realização esporádica, por este ser um dos vários locais para verificação do pulso e nem sempre o mais usual.

Ressalta-se que neste estudo nenhum dos itens relacionados foi citado como nunca realizado por mais de $50 \%$ dos enfermeiros. Dentre os itens assim mencionados por menos do que $50 \%$ deles, destacaram-se os seguintes: pressão intracraniana $(38,5 \%)$, altura $(26,9 \%)$, linfonodos $(23,1 \%)$, mamas $(19,2 \%)$, pesquisa de estruturas internas $(19,2 \%)$ e exame das articulações $(19,2 \%)$.

Além dos 45 itens do exame físico relacionados no instrumento proposto possibilitou-se a inclusão de outros pelos enfermeiros. Analisando-se a totalidade das sugestões apresentadas considerou-se que apenas duas eram pertinentes (amplitude de pulso e avaliação da fontanela), pois os sugeridos (lesões cutâneas e cianose, prótese dentária, acuidade visual e auditiva e simetria de tórax) encontravam-se já contemplados no instrumento. Foram ainda sugeridos pelos enfermeiros, itens relativos às drenagens gástricas, torácicas, urinárias, incisionais e a outras medidas diagnósticas e terapêuticas.

Essa diversidade de sugestōes retrata a dificuldade de se estabelecer limites bem definidos entre o que deve ou não ser incluido no exame físico. Não se pode perder de vista que ele é apenas uma das formas de obtenção de 
dados para a análise das condições físicas do paciente. Devem ser consideradas outras fontes de igual importáncia como entrevista, exames laboratoriais, relatórios de outros profissionais e a observação dos artefatos e procedimentos terapeuticos utilizados na assistência ao paciente.

BATES (1982) afirma que há necessidade de outros achados clínicos e exames laboratoriais para uma abordagem relativamente abrangente dos pacientes. Essa mesma autora considera ainda que não existe nenhum exame físico adequado claramente definido e que para torná-lo mais completo devese direcioná-lo para os aspectos específicos de cada paciente tais como idade, sexo e sintomas. Reconhece-se também que os próprios achados no exame físico de um paciente podem apontar para a necessidade de maior exploração das alteraçōes.

Pode-se dizer que os resultados obtidos no presente estudo, dão indícios de que os itens freqüentemente realizados pela maioria dos enfermeiros de UTI caracterizam de certa forma, o exame físico mais genérico do paciente crítico. No entanto, as especificidades deste exame podem estar presentes nos itens que surgiram em menor freqüência ou que se destacaram como esporadicamente ou nunca realizados.

c. Ensino do exame físico

Quanto ao ensino dos itens do exame físico, verificou-se que a totalidade deles (45 itens) foi citada como sendo de responsabilidade dos cursos de graduação por, no mínimo, $69,2 \%$ dos enfermeiros. Cabe ressaitar que os itens: avaliação do ritmo cardíaco pela ausculta e da pressão intracraniana foram os que apareceram com menor percentual de indicaçào para ensino nesse nível.

Em nível de pós-graduação "sensu stricto" não foi atribuída qualquer responsabilidade pelo ensino do exame físico. Entretanto, constatou-se que do total de 45 itens, 27 receberam indicação de que o seu ensino fosse de responsabilidade de cursos de atualização e especialização, caracterizados no presente trabalho como cursos extracurriculares.

O QUADRO III especifica os 9 itens que receberam, no mínimo, cinco indicações para o ensino nesse nível.

QUADRO III - Itens do exame fisico cuia responsabilidade pelo ensino foi atribuida aos cursos extracurriculares (atuauzação e especializaçāo). São Paulo, 1992.

\begin{tabular}{lcr}
\hline Itens & $\mathrm{N}$ & $\%$ \\
\hline Pressão intracraniana & 12 & 46,2 \\
Respiração - ruidos (ausculta) & 10 & 38.5 \\
Abdômen - pesquisa de estruturas internas & 10 & 38,5 \\
Ausculta cardíaca - ritmo & 9 & 34,6 \\
Ausculta cardiaca - frequeència & 7 & 26.9 \\
Nivel de consciència & 6 & 23,1 \\
Exame pupilar & 5 & 19,2 \\
Abdômen - pesquisa de ascite & 5 & 19,2 \\
Pescoço - linfonodos & 5 & 19.2 \\
\hline
\end{tabular}


Considerando o exame físico como a base para o desenvolvimento da atividade clínica do enfermeiro, reiteram-se os resultados encontrados no presente estudo quanto à responsabilidade dos cursos de graduaçāo no ensino deste conteúdo.

Em estudo junto a educadores de enfermagem, SOLOMON (1990) verificou que existe concordancia no fato de que as habilidades para a avaliação física são a base para o processo de enfermagem e devem portanto, ser introduzidas precocemente durante a graduação.

A definição do momento apropriado para iniciar o ensino do exame físico não suscita muitas divergências. Porém, persistem dúvidas relativas tanto à organização e progressão do conteúdo no decorrer da graduação, quanto à sua continuidade e aprofundamento em cursos extracurriculares. Corroborando essa situação os dados do QUADRO III mostram que existem enfermeiros que atribuem a responsabilidade do ensino de alguns itens do exame físico aos cursos extracurriculares. Torna-se dificil analisar-possiveis justificativas para essas. respostas diante da multiplicidade de fatores a serem considerados quando se trata de determinar a localização do ensino de cada item do exame físico.

$O$ entendimento dos respondentes sobre quais sejam as finalidades dos cursos nos diferentes niveis pode ser um dos fatores que interferem nessa determinação. Outros fatores poderiam estar relacionados à prática protissional de cada pessoa ou grupo. Entre esses fatores incluem-se: a especificidade da clientela atendida, a percepção de lacunas no conhecimento e experiência já adquiridos e a utilidade dos dados obtidos com o exame físico para a prática profissional.

Esses aspectos certamente não esgotam todas as possibilidades de análise mesmo porque a percepção da complexidade das técnicas envolvidas no exame físico e a atribuição de significado aos dados obtidos passa necessariamente por questões de ordem individual, sobretudo motivação para o desenvolvimento profissional.

Em resumo, năo há dúvidas de que o ensino do exame físico deva ser feito na graduação e de que não existe consenso no que se refere a abrangència do conteúdo desse ensino a esse nível e o que deveria ser destinado a outros niveis de formação. No entanto a efetividade do aprendizado ao nivel de graduação pode ser questionado pelos dados da TABELA 1 que mostram em que momento o mesmo ocorreu para os enfermeiros participantes deste estudo.

TABELA 1 - Momentos de maior significado para os enfermeiros no aprendizado do exame físico. São Paulo, 1992.

\begin{tabular}{lrrr}
\hline Momentos & N & & \\
\hline Prática profissional & 11 & $32,3 \%$ & \\
Cursos extracurriculares & 9 & $26,5 \%$ & $52,9 \%$ \\
Treinamento institucional & 7 & $20,6 \%$ & $47.1 \%$ \\
Curso de graduação & 7 & $20,6 \%$ & \\
\hline TOTAL & 34 & $100,0 \%$ & \\
\hline
\end{tabular}


Pela TABELA 1 verifica-se que $32,3 \%$ das respostas recairam sobre o aprendizado do exame físico na prática profissional, sendo indicados os cursos extracurriculares por $26,5 \%$ das respostas. Ainda, o treinamento institucional e o curso de graduação foram apontados por $20,6 \%$ das respostas, como momentos de aprendizado do exame físico.

Conforme já mencionado, a responsabilidade pelo ensino de todos os itens do exame físico foi atribuída aos cursos de graduação pela maioria dos enfermeiros $(69,2 \%)$. Porém os dados da TABELA 1 mostram que na experiência da maioria dos enfermeiros esse não foi o momento mais significativo em que o aprendizado se deu. As respostas indicaram que a prática profissional foi o momento de maior importância, com $32,3 \%$ das respostas.

Sem ignorar as dificuldades conceituais sobre o processo ensino-aprendizagem, a continuidade na prática profissional é indiscutível, embora não seja considerada uma instância formal de ensino.

A contradição entre o que esses enfermeiros consideram que "deve ser" e a sua experiência, suscita vários questionamentos que este trabalho não pretende esclarecer, mas pontua para reflexão: o conteúdo do exame físico oferecido nos cursos de graduação não atende às demandas do exercício profissional? Ainda prevalece a idéia de que as escolas devem assumir para si a responsabilidade plena pela formação profissional?

Os dados mostram, por um lado, que o maior aprendizado ocorreu na prática profissional quando comparado ao curso de graduação. Por outro lado, agrupando-se a prática profissional com o treinamento institucional e os cursos de graduaçāo com os extracurriculares verifica-se uma diferença entre o aprendizado adquirido na instituição de trabalho $(52,9 \%)$ e na instituição de ensino $(47,1 \%)$. Embora discreta, essa diferença pode indicar que quando se trata dos momentos nos quais o aprendizado é mais significativo parece ser inegável o impacto da prática profissional. Esse resultado pode ser explicado ao considerar-se que é na prática que o aprendizado se consolida e se reveste de maior significado. Apesar disso, deve-se questionar se o conteúdo do exame físico oferecido nos cursos de graduação tem atendido às demandas básicas do exercício profissional. Esse questionamento nos remete aos resultados referentes à responsabilidade do ensino do exame físico que foi atribuída principalmente aos cursos de graduaçāo.

\section{CONCLUSÕES}

Os resultados obtidos neste estudo permitiram as seguintes conclusões:

- em relação a periodicidade de realização dos itens componentes do exame físico, verificou-se que do total de 45 itens apresentados, 31 $(68,9 \%)$ eram realizados freqüentemente, $5(11,1 \%)$ esporadicamente, sendo que nenhum dos itens foi citado como nunca realizado, considerando em todas estas freqüências as respostas fornecidas por mais de $50 \%$ dos enfermeiros; 
- no que se refere a fase de formação mais adequada para o ensino dos itens do exame fisico, constatou-se que esta responsabilidade foi atribuida ao curso de graduação por $18(69,2 \%)$ dos 26 enfermeiros respondentes;

- quanto ao momento mais significativo de aprendizado do exame físico, verificou-se que $32,3 \%$ das respostas dos enfermeiros recaíram sobre a prática profissional seguida pelos cursos extracurriculares, com $26,5 \%$ das respostas.

Apesar dos objetivos do presente estudo terem sido alcançados, cabe ressaltar que algumas limitações foram evidenciadas no decorrer do mesmo, mostrando a necessidade de aprofundar a análise dos fatores que foram apenas mencionados na discussão dos resultados. Além disso, deve-se considerar que o próprio tamanho da população se constituiu num fator limitante para a consideração mais genérica destas conclusões.

Esclarece-se, ainda, que este trabalho representa uma parte inicial de um estudo mais abrangente sobre a prática do exame físico pelos enfermeiros de UTI.

KIMURA, $M$ et al. The physical assessment and the ICU nurse. Rev. Esc. Enf. USP, v.28. n.2, p. 156-70, aug. 1994.

The goal of this study was to analyse some practicing, teaching and learning aspects of physical examination done by ICU's nurses. It was accomplished with 26 ICU nurses that concluded the Intensive Care Nursing Specialization Course at the School of Nursing at Sāo Paulo University. The results showed that 31 (68.9\%) of the 45 presented itens were done frequently by more than $50 \%$ of the nurses. The professional practice was considered the most important moment to physical examination learning. The responsability by teaching was attributed to undergraduation course by $69,2 \%$ of the nurses.

UNITERMS: ICU - physical examination - nursing teaching

\section{REFERÊNCIAS BIBLIOGRÁFICAS}

BATES. B.: LYNAUGH. J. Laying the foundations for medical nursing practice. Am.J.Nurs. . v.73, n.8. p.1375-9, 1973.

BATES. B.: LYNAUGH, J. Teaching physical assessment. Nurs.Outlook , v.23, n.5, p.297$302,1975$.

BATES, B. Propedeutica médica. Rio de Janeiro, Interamericana, 1982.

COLWELL, C.B.; SMITH. J. Determining the use of physical assessment skills in the clinica! setting. J.Nurs.Educ, v.24, n.8, p.333-9, 1985. 
HORTA, W.A. A observação sistematizada na identificação dos problemas de enfermagem em seus aspectos físicos. Rio de Janeiro, 1968,60p. Tese(Livre-Docência) - Escola de Enfermagem Ana Neri, Universidade Federal do Rio de Janeiro.

JACKSON, B.S.; MANTLE, D.D. Teaching patient assessment: the pros \& cons of clinical rounds. J.Nurs.Educ, v.16, n.2, p.24-9, 1977.

MORGAN, W.L.; BATES, B. Should nurses do physical examinations? Ann.Int.Med, , v.75, n.2, p.314-5, 1971.

SOLOMON, J. Physical assessment skills in undergraduate curricula. Nurs.Outlook , v.38, n.4, p.194-5, 1990. 


\section{ANEXO I \\ QUESTIONÁRIO}

$N^{\circ}$

\section{Dados Gerais}

Idade Sexo Tempo de Formatura

Tempo de trabaiho em UTI

Caracterizaçāo da UTI: - $\mathrm{N}^{\circ}$ de leitos

- Tipo de atendimento: geral especializado

- Tipo de paciente: pediátrico adulto clínico cirúrgico

II. Dados específicos

1. Dentre as alternativas abaixo indique aquela em que o seu aprendizado sobre o exame físico foi mais significativo: no decorrer do curso de graduação em curso especifico, durante o treinamento na instituiçāo em cursos extracurriculares sem curso, no decorrer da prática profissional outro

Justifique:

Responda as 4 perguntas seguintes no Quadro de Respostas em anexo de acordo com as instituiçōes relativas a cada questāo.

2. Com que freqüência você realiza os itens do exame físico listados no Quadro?

Observação: para responder a esta questão procure considerar tanto o número de vezes que realiza cada item num dia como também a periodicidade que os utiliza. Empregue a legenda abaixo na coluna correspondente $(\mathcal{Z})$

F - Freqüentemente

E - Esporadicamente

N - Nunca

3. Quanto a realizaçào de cada item do exame físico contribui para a determinação de condutas de enfermagem?

Observação: Utilize a legenda abaixo, na coluna correspondente (3) 

M - muito
P - Pouco
N - Nada

4. Que barreiras ou dificuldades você encontra para a realização dos diferentes itens do exame fissico?

Observação: Classifique conforme as alternativas apresentadas abaixo. Caso haja mais de uma opção em cada item coloque-as em ordem de prioridade, na coluna (4)

Legendas - Barreiras/ Dificuldades
A. Nenhuma
B. Falta de equipamento
C. Presença de estagiários
D. Falta de familiaridade na execução do item
E. Nāo é responsabilidade da enfermeira
F. Não é identificado como área problemática da clientela
G. Outros. Especifique:
$\mathrm{G}_{1}$

$\mathrm{G}_{2}$

$\mathrm{G}_{3}$

5. Em que nivel da formaçāo do enfermeiro você considera que os itens do exa. me físico devam ser ensinados?

Observaçào: Escolha a melhor alternativa levando em conta a principal responsabilidade pelo ensino do item dentre os diferentes niveis de formaçāo.

Utilizar a legenda abaixo, na coluna (5)

G - Graduação

A - Atualização

E - Especializaçāo

PG - Pós-graduaçāo (mestrado/doutorado)

\begin{tabular}{|c|c|c|c|}
\hline \multicolumn{4}{|c|}{ QUADRO DE RESPOSTAS } \\
\hline $\begin{array}{l}\text { Tópicos } \\
\text { Itens }\end{array}$ & frequência (2) & $\begin{array}{r}\text { contribuição } \\
\text { p/a conduta (3) }\end{array}$ & barreiras (4) $\left|\begin{array}{r}\text { responsabilidad } \\
\text { e pelo ensino } \\
15)\end{array}\right|$ \\
\hline $\begin{array}{l}\text { Peso } \\
\text { Altura } \\
\text { Temperatura }\end{array}$ & & & 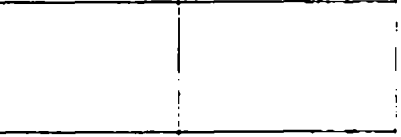 \\
\hline $\begin{array}{l}\text { Respiraçào } \\
\text { Frequiência } \\
\text { amplitude } \\
\text { ritmo } \\
\text { simetria }\end{array}$ & & & 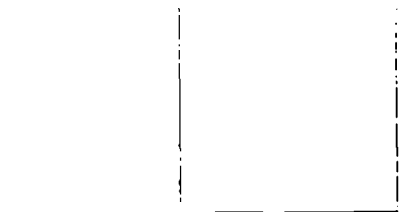 \\
\hline
\end{tabular}




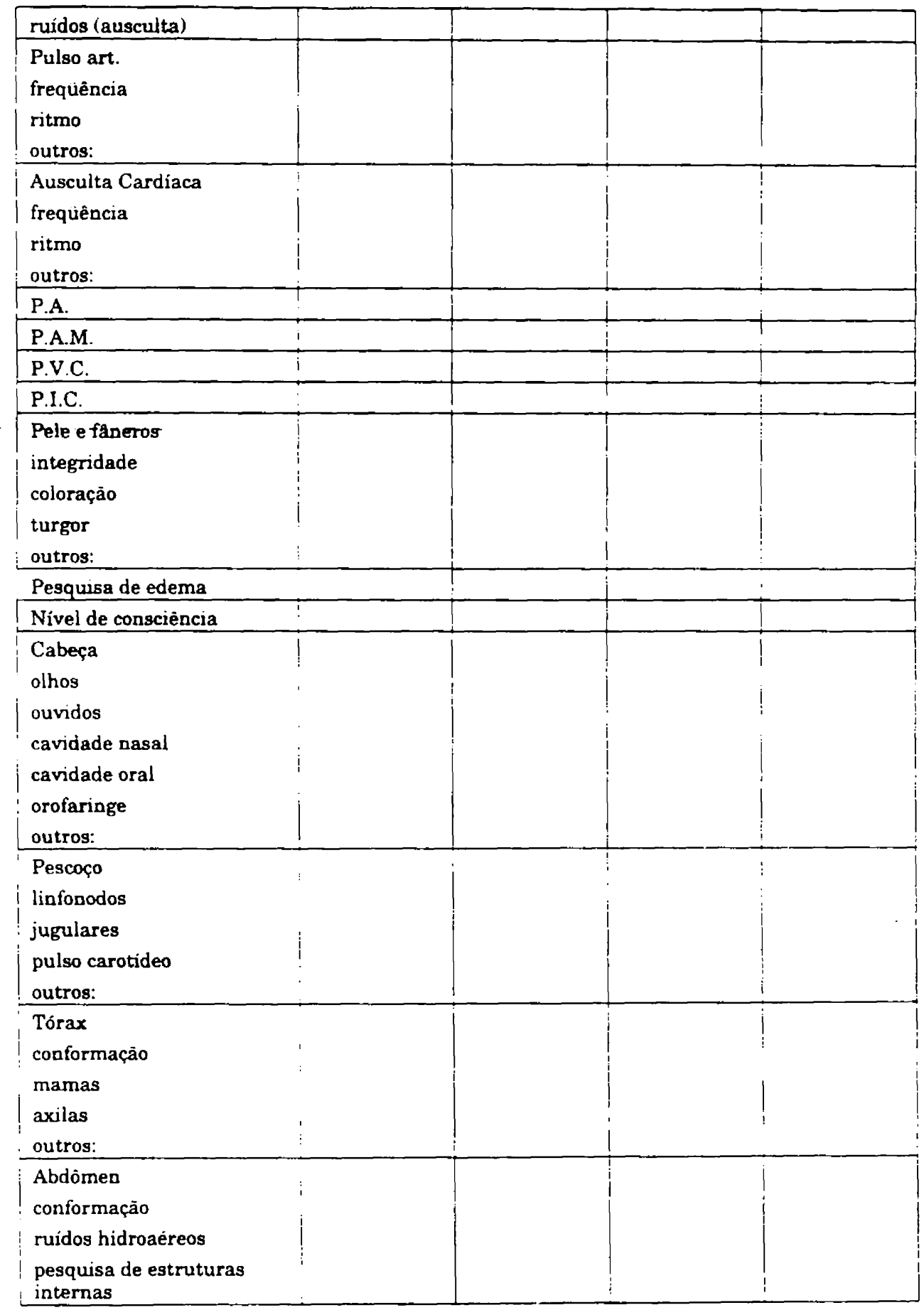




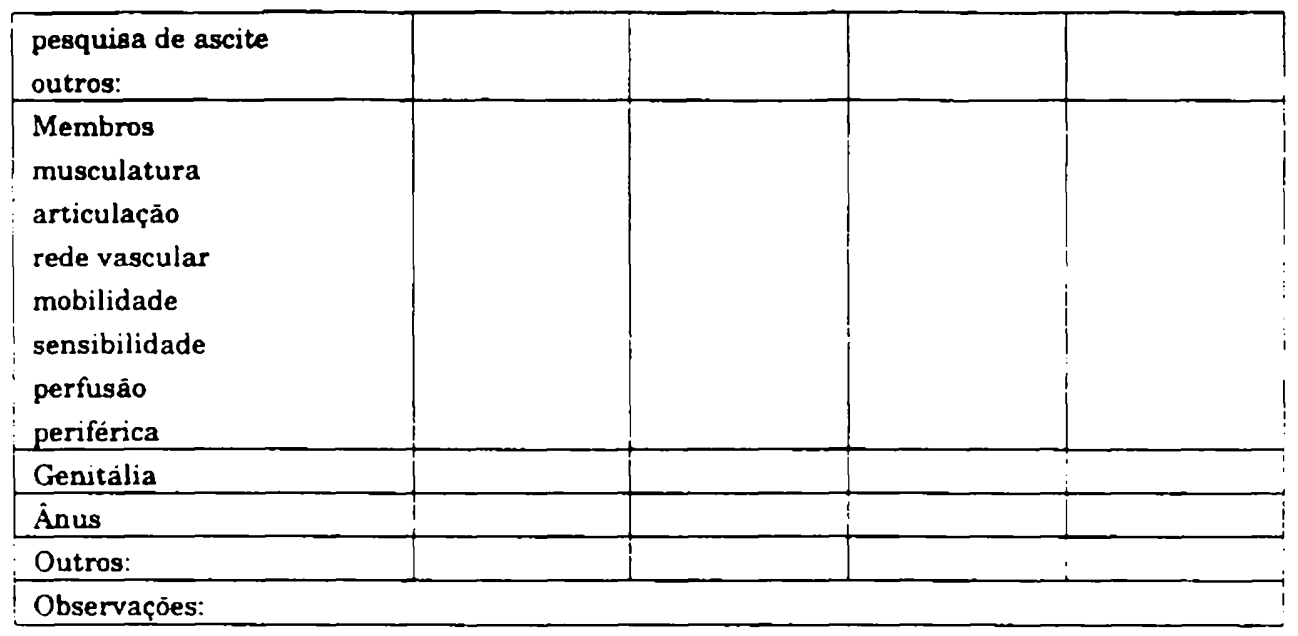

